\title{
Feedback-Controlled Topological Reconfiguration of Molecular Assemblies for Programming Supramolecular Structures
}

\author{
Panpan Li, ${ }^{1}$ Aixin Song, ${ }^{2}$ Jingcheng Hao, ${ }^{2}$ and Xu Wang ${ }^{*}, 1,2$ \\ ${ }^{1}$ National Engineering Research Center for Colloidal Materials, School of Chemistry and \\ Chemical Engineering, Shandong University, Jinan, Shandong, 250100, China \\ ${ }^{2}$ Key Laboratory of Colloid and Interface Chemistry of the Ministry of Education, School of \\ Chemistry and Chemical Engineering, Shandong University, Jinan, Shandong, 250100, China \\ *Email: wangxu@sdu.edu.cn
}




\begin{abstract}
In biology, nonequilibrium assembly is characterized by fuel-driven switching between associating and nonassociating states of biomolecules. This dynamic assembly model has been used routinely to describe the nonequilibrium processes in synthetic systems. Here, we present a G-quartet-based nonequilibrium system mediated by fuel-driven co-assembly of guanosine 5'-monophosphate disodium salt hydrate and urease. Addition of lanthanum(III) ions to the system caused macroscopic dynamic switching between precipitates and hydrogels. Interestingly, combined analyses of the nonequilibrium systems demonstrated that molecules could switch between two distinct associating states without undergoing a nonassociating state, suggesting a nonequilibrium assembly mechanism of topological reconfiguration of molecular assemblies. We detailed quantitatively the nonequilibrium assembly mechanism to precisely control the phase behaviors of the active materials and, therefore, were able to apply the materials for transient-gel-templated polymerization and transient circuit connection. This work presents a new nonequilibrium system with unusual phase behavior, and the resultant active hydrogels hold substantial promise in applications including fluid confinements and transient electronics.
\end{abstract}




\section{Introduction}

Nonequilibrium assembly occurs widely in biological systems. With continuous energy input, activated building blocks assemble specifically into transient structures that subsequently disassemble into individual molecules when fuel or energy is depleted. ${ }^{1-5}$ Living organisms use the switching of biomolecules between associating and nonassociating states to construct sophisticated active structures that respond to multiple stimuli and exert complex functions. ${ }^{6}$ Mimicking the rules of nonequilibrium assembly in nature, chemists have constructed nonequilibrium systems that are fuel-driven, transient, and far from thermodynamic equilibrium. ${ }^{6-17}$ This research field has developed from dissipative structure design towards advanced function exploration, and the field has achieved rich results such as the applications of nonequilibrium chemistry for controlled drug release and information encryption. ${ }^{10,11,} 14$ Recently, our group reported the use of nonequilibrium chemistry to regulate the transient healing ability of thermodynamically stable and kinetically inert polymer hydrogels. ${ }^{18-22}$ This regulation struck the right balance between kinetic stability and intrinsic healing ability of polymer materials. Nevertheless, comprehensive research is still lacking for nonequilibrium assembly mechanisms in synthetic systems.

Unlike site-specific binding with high affinity in biological systems, nonequilibrium assembly in artificial systems is controlled mostly by supramolecular interactions between nonspecific binding sites. ${ }^{8,} 15$ Therefore, the nonequilibrium assembly models in artificial systems, in principle, can be diversified. So far, most studies used routinely the conventional 
assembly-disassembly mechanism to describe the nonequilibrium assembly processes, and very few nonequilibrium assembly models beyond the conventional mechanism have been clearly proposed. George and coworkers presented a multistate transient self-assembly mechanism and verified the nonequilibrium assembly model in an adenosine-phosphate-fueled supramolecular polymer system. ${ }^{12}$ The multistate transient self-assembly may provide a highly modular control over system properties. In the other example, Hermans and colleagues demonstrated the supramolecular oscillations, travelling fronts and patterns in a perylene-diimide-derivative-based system, bringing us closer to the creation of life-like materials. ${ }^{13}$ Here, we described a nonequilibrium assembly model of feedback-controlled topological reconfiguration of molecular assemblies. This model was validated in a co-assembling system containing guanosine 5'-monophosphate disodium salt hydrate (GMP) and urease. The macroscopic dynamic sol-to-gel-to-sol transitions generated by the microscopic nonequilibrium co-assembly of GMP and enzyme molecules could be magnified to yield precipitate-to-gel-to-precipitate transitions by adding lanthanum(III) ions $\left(\mathrm{La}^{3+}\right)$. The resultant macroscopic transient hydrogels can serve as self-erasable wells and self-dissociative ionotronics because of their good injectability, ionic conductivity, and dissipative properties.

\section{Results}

GMP has unique self-assembly behaviors under acidic conditions because of the formation of G-quartet and G-quadruplexes. ${ }^{23-27}$ The phosphate groups of GMP endow the molecule with $\mathrm{pH}$ responsiveness and ion binding capacity. ${ }^{26}$ The rational integration of GMP with a fuel-driven 
$\mathrm{pH}$ clock is promising for building a distinctive nonequilibrium system. The autocatalytic urea-urease reaction is a classic $\mathrm{pH}$ clock, and it has been widely used for controlling the properties of supramolecular systems. ${ }^{28,}{ }^{29}$ We also used this $\mathrm{pH}$ clock to balance the kinetic stability and healing ability of acylhydrazone-based polymer hydrogels in the previous work. ${ }^{20}$ Here we coupled GMP with urease to construct two $\mathrm{pH}$ feedback systems without and with $\mathrm{La}^{3+}$, namely, GMP/urease and GMP/La/urease systems (Scheme 1). The urease molecules not only served as building blocks to participate in the construction of supramolecular structures but also acted as biocatalysts to regulate the nonequilibrium assembly processes. These co-assembling systems exhibited different self-assembly behaviors at different $\mathrm{pH}$ (Fig. S1). We used methyl red, a $\mathrm{pH}$ indicator with two color-changing $\mathrm{pH}$ values of 4.4 and 6.2 (from red to orange to yellow), to monitor the $\mathrm{pH}$-induced phase transitions in the GMP/urease and GMP/La/urease systems (Fig. S2). We found that the phase transition behaviors of the systems did not change after adding a small amount of methyl red. Upon addition of chemical fuels (i.e., acidic urea solutions) to the GMP/urease system at initial $\mathrm{pH}$ of 8, a fast sol-to-gel transition occurred (Fig. 1a) because of the rapid decline of $\mathrm{pH}$; this sol-to-gel transition was accompanied by an alteration of the self-assembled structures from irregular shaped nanoparticles to 1D nanofibers (Fig. 1b) as observed by cryogenic transmission electron microscopy (cryo-TEM). Then, the system slowly recovered to a sol state because of the $\mathrm{pH}$ increase caused by the biocatalytic generation of ammonia, along with the gradual dissociation of the nanofibers and the formation of irregular shaped aggregates. The Tyndall effect (Fig. 1b and S3) further confirmed that the 
supramolecular aggregates in the GMP/urease system existed in both the sol and gel states. The introduction of $\mathrm{La}^{3+}$ to the GMP/urease system caused further aggregation, and a precipitate-to-gel-to-precipitate transition was observed (Fig. 1a). Compared with the GMP/urease system, the $\mathrm{La}^{3+}$-containing system had more apparent structural evolution at both microscopic and macroscopic levels and possessed a longer cycle of nonequilibrium assembly (Fig. 1a, b). We ascribed these properties to the combined effect of ion-dipole and electrostatic interactions between GMP, $\mathrm{La}^{3+}$, and/or urease. Fig. 1c shows that the lifetime of the biocatalytic feedback-driven transient supramolecular hydrogels could be tuned simply by varying the urea concentration in the chemical fuels. This tuning was possible mainly because urease was still active in the systems (Fig. S4), and the enzymatic reaction rate increased with increasing urea concentration in a certain range (Fig. 1d). The data fitted to the Michaelis-Menten equation ${ }^{30}$ yielded a maximum velocity $\left(V_{\max }\right)$ and a Michaelis constant $\left(K_{\mathrm{m}}\right)$ of $6.2 \pm 0.3 \mathrm{mM} / \mathrm{min}$ and 34.9 $\pm 6.2 \mathrm{mM}$, respectively. These results indicated that the selected urea concentrations were within an adjustable range. 

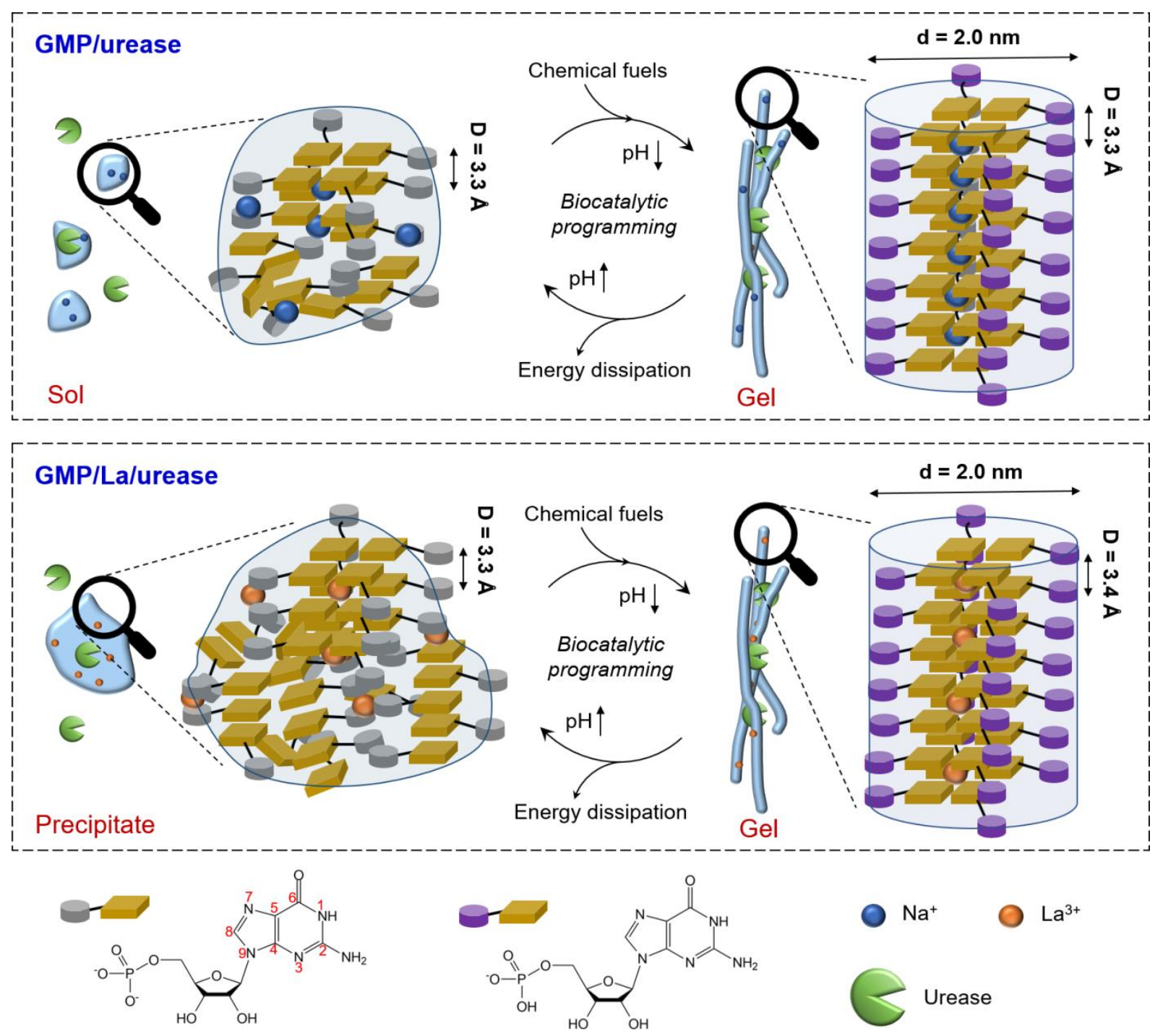

Scheme 1 Nonequilibrium assembly in the GMP/urease and GMP/La/urease systems. 

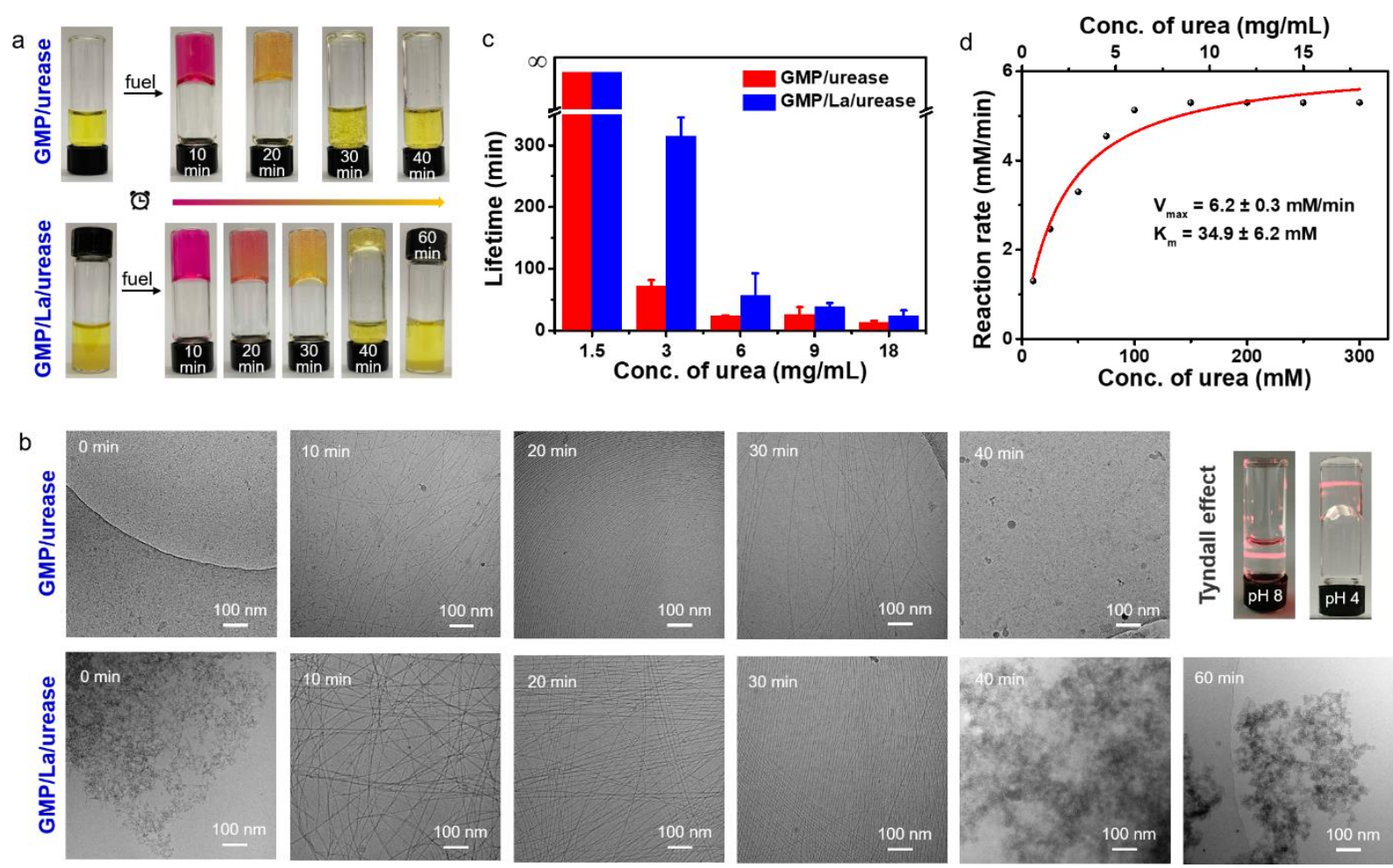

Fig. 1 (a) Photographs showing the sol-to-gel-to-sol and precipitate-to-gel-to-precipitate transitions of GMP/urease and GMP/La/urease systems. Methyl red was used as a $\mathrm{pH}$ indicator. (b) Cryo-TEM images of GMP/urease and GMP/La/urease systems at different times after fuel addition; the $0 \mathrm{~min}$ is the condition before fuel addition. The photographs at the top right of (b) show the Tyndall effect of GMP/urease systems at $\mathrm{pH} 8$ and $\mathrm{pH} 4$. (c) Lifetimes of the transient hydrogels driven by the addition of chemical fuels with different urea concentrations. (d) The Michaelis-Menten curve describing the influence of urea concentration on enzymatic reaction rate.

To study the assembly mechanism, we characterized the nanostructures of the supramolecular aggregates at $\mathrm{pH} 8$ and $\mathrm{pH} 4$, which corresponded to the starting/ending point (sol or precipitate state) and an important intermediate point (gel state) of the dissipative assembly processes, respectively. All the X-ray diffraction (XRD) patterns for the dry samples of GMP/urease and GMP/La/urease at $\mathrm{pH} 8$ and $\mathrm{pH} 4$ showed a characteristic peak at approximately $27^{\circ}$ (Fig. 2a). This peak demonstrated that the GMP molecules in all the systems 
existed in an associating state. Interestingly, the samples of GMP/urease and GMP/La/urease at $\mathrm{pH} 4$ had a peak at $q=3.1 \mathrm{~nm}^{-1}$ in the small-angle X-ray scattering (SAXS) patterns (Fig. 2b). The correlated $d$ value of $2.0 \mathrm{~nm}$ was consistent with the diameter of columnar G-quadruplexes formed by stacked G-quartets. ${ }^{26}$ By contrast, the samples of GMP/urease and GMP/La/urease at pH 8 did not show any characteristic peaks in the SAXS patterns, which implied that incomplete G-quadruplexes or no G-quadruplexes existed in these systems. We used Fourier-transform infrared (FTIR) spectroscopy to clarify the influence of system $\mathrm{pH}$ on molecular assembly behavior. The results in Fig. $2 \mathrm{c}$ showed that the peak at $1678 \mathrm{~cm}^{-1}$ assigned to the stretching vibration of $\mathrm{C}(6)=\mathrm{O}$ groups of GMP shifted to higher wavenumbers after the addition of urease and/or $\mathrm{La}^{3+}$; the shift to higher wavenumbers was ascribed to the supramolecular interactions between GMP, urease, and/or metal ions. At the same time, the peak at $1490 \mathrm{~cm}^{-1}$ that belonged to the stretching vibration of $\mathrm{N}(7)-\mathrm{C}(8)$ in GMP shifted to lower wavenumbers because of the formation of hydrogen bonds between the contiguous guanine skeletons. The peaks at 1069 and $978 \mathrm{~cm}^{-1}$ were attributed to the antisymmetric and symmetric stretching vibrations of the phosphate groups of GMP, respectively. Significant differences in peak shape at 1069 and 978 $\mathrm{cm}^{-1}$ were found between $\mathrm{pH} 8$ and $\mathrm{pH} 4$ in both GMP/urease and GMP/La/urease systems. These differences were likely the result of different degrees of protonation of the GMP phosphate groups at different $\mathrm{pH}$ (Scheme 1). The combined XRD, SAXS and FTIR spectra analyses of GMP/urease and GMP/La/urease systems confirmed that the molecular aggregation states at the starting/ending point and at the important intermediate point were completely 
different. Notably, at the same GMP concentration $(50 \mathrm{mM})$, the GMP/urease system formed hydrogels at $\mathrm{pH} 4$, whereas the GMP system without urease was a typical colloidal solution (Fig. S5, S6). This fact suggested that urease can participate in the molecular assembly and contribute to the formation of $3 \mathrm{D}$ network structures. To quantitatively analyze the amount of urease involved in molecular assembly, we labeled urease with fluorescein isothiocyanate (FITC-urease), and the amounts of free FITC-urease and FITC-urease in molecular assembly were determined by ultraviolet-visible spectrophotometry (Fig. 2d). We found that, for the GMP/La/urease system, over $80 \%$ of the urease participated in molecular assembly at pH 4 , which was much greater than that at $\mathrm{pH} 8$ (Fig. 2e). This difference was mainly attributed to the stronger electrostatic interactions between positively charged urease and negatively charged GMP at pH 4 (Fig. S7). Next, we used the fluorescence spectroscopy to investigate the binding affinity between GMP and urease. ${ }^{31,32}$ The intrinsic fluorescence of urease is substantially provided by tryptophan (Trp). Hence, the information related to the urease-GMP interactions and GMP-induced conformational change around the binding site could be provided by the fluorescence intensity change of Trp. The maximum emission wavelength of urease was $339 \mathrm{~nm}$, and the fluorescence intensity of urease decreased with increasing GMP concentration at $\mathrm{pH} 8$ and $\mathrm{pH} 4$ (Fig. 2f, g). The fluorescence quenching could be caused by the binding between GMP and urease. The fluorescence quenching mechanism of urease induced by GMP was investigated by the Stern-Volmer analysis (Fig. S8). The Stern-Volmer curve was linear when the GMP concentration ranged from 0 to $0.1 \mathrm{mM}$. The dynamic quenching constants $\left(K_{\mathrm{SV}}\right)$ calculated from 
the slopes were $1.14 \times 10^{4}$ and $7.79 \times 10^{3} \mathrm{M}^{-1}$ for $\mathrm{pH} 8$ and $\mathrm{pH} 4$, respectively. Hence, the bimolecular quenching rate constants $\left(K_{\mathrm{q}}\right)$ calculated from the average lifetimes of the biomolecules without quencher $\left(\tau_{0}\right.$, typically $\left.10^{-8} \mathrm{~s}\right)$ were $1.14 \times 10^{12}$ and $7.79 \times 10^{11} \mathrm{M}^{-1} \mathrm{~s}^{-1}$ for $\mathrm{pH} 8$ and $\mathrm{pH} 4$, respectively. These values were greater than the maximum scatter collision quenching constant of various quenchers with the biomacromolecule $\left(2.0 \times 10^{10} \mathrm{M}^{-1} \mathrm{~s}^{-1}\right)$ for dynamic quenching. This result indicated that quenching was initiated by static quenching as a result of the formation of urease-GMP complexes. The binding constants $\left(K_{\mathrm{a}}\right)$ between GMP and urease were $3.05 \times 10^{4}$ and $8.05 \times 10^{4} \mathrm{M}^{-1}$ for $\mathrm{pH} 8$ and $\mathrm{pH} 4$, respectively, obtained from the double logarithm regression curves (Fig. 2h). These results demonstrated that there was a stronger binding force between GMP and urease at $\mathrm{pH} 4$ than that at $\mathrm{pH}$. The distribution of FITC-urease in GMP/La/FITC-urease hydrogels at pH 4 was assessed by confocal laser scanning microscopy (CLSM). For precisely locating the hydrogels, we added benzothiazole dye thioflavin T (ThT), a fluorescent molecule capable of detecting the formation of G-quadruplexes by fixing the chromophoric skeleton ${ }^{26}$ (Fig. 2i). The morphology of the GMP/La/FITC-urease hydrogel was clearly observed (Fig. $2 \mathrm{j}$, red) because of the efficient implant of ThT molecules in the G-quadruplex structures. The fluorescence of FITC-urease was strong and uniform (Fig. 2j, green), and the large orange areas in the merged image illustrated that the FITC-urease molecules distributed evenly in the hydrogel (Fig. 2j). 

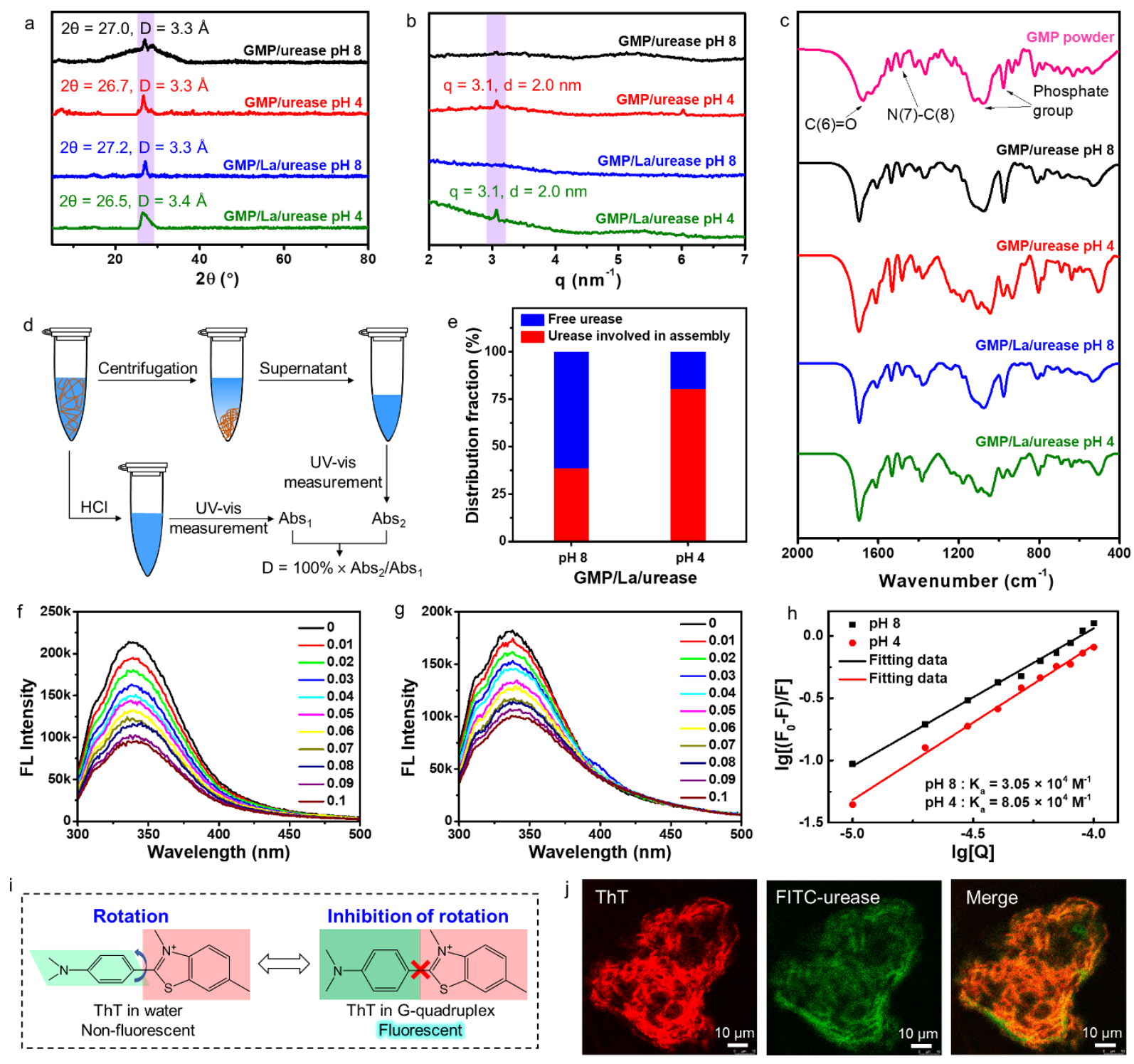

Fig. 2 (a, b) XRD (a) and SAXS (b) patterns of GMP/urease and GMP/La/urease systems at pH 8 and $\mathrm{pH}$ 4. (c) FTIR spectra of GMP powder, GMP/urease and GMP/La/urease systems at $\mathrm{pH} 8$ and $\mathrm{pH}$ 4. (d) Schematic of urease distribution measurements in GMP/La/urease systems. (e) Distribution of urease in GMP/La/urease systems at $\mathrm{pH} 8$ and pH 4. (f, g) Effects of GMP (0 $0.1 \mathrm{mM})$ on the fluorescence spectra of urease at $\mathrm{pH} 8$ (f) and $\mathrm{pH} 4(\mathrm{~g})\left(\lambda_{\mathrm{ex}}=280 \mathrm{~nm}\right)$. (h) Double-lg plot of GMP quenching effect on the fluorescence of urease. (i) Chemical structure and fluorescence enhancing mechanism of ThT. (j) CLSM images of ThT-containing GMP/La/FITC-urease hydrogels at $\mathrm{pH} 4$.

The fluorescence enhancing property of ThT was also used to monitor the structural evolution of the molecular assemblies during the nonequilibrium assembly process. After fuel 
addition, both GMP/urease and GMP/La/urease systems showed an initial increase in fluorescence intensity, followed by a decline in intensity (Fig. 3a, b). These results suggested that the molecules in the nonequilibrium systems temporarily accessed an associating state with a higher degree of order. And the followed decreased ThT fluorescence intensity indicated the dissociation of G-quadruplex structures. ${ }^{33}$ Moreover, stronger fluorescence intensity was observed in the GMP/La/urease system, which affirmed the positive effect of $\mathrm{La}^{3+}$ in constructing G-quadruplex structures. The time-dependent fluorescence properties of the ThT-containing nonequilibrium systems suggested that our transient supramolecular assemblies had the potential to be used as fluorescence modulators. ${ }^{34}$ Circular dichroism (CD) spectroscopy was used to monitor the evolution of G-quadruplex structures in the GMP/urease and GMP/La/urease systems. Before the addition of chemical fuels, we did not observe a characteristic signal in the CD spectra for these two systems (Fig. 3c, d and S9). After fuel addition, the appearance of a positive peak around $240 \mathrm{~nm}$ and a negative peak around $276 \mathrm{~nm}$ in the CD spectra revealed the formation of G-quadruplex structures, in which the G-quartets were stacked in an antiparallel fashion. ${ }^{26,35}$ The CD signals then gradually weakened, which suggested the dissociation of the G-quadruplex structures. Notably, although the G-quadruplex structures dissociated, the GMP molecules always existed in an associating state, as revealed by the cryo-TEM (Fig. 1b) and XRD (Fig. 2a). The time-dependent fluorescence and CD results (Fig. 3a-d) were in reasonable agreement with the macroscopic and microscopic structure characterizations in Fig. 1a, b. Although the hydrogel was becoming weaker due to waste 
accumulation, repeated sol-to-gel-to-sol and precipitate-to-gel-to-precipitate transitions for the GMP/urease and GMP/La/urease systems could still be achieved, respectively, by refueling the systems with acidic urea solutions (Fig. 3e, f).
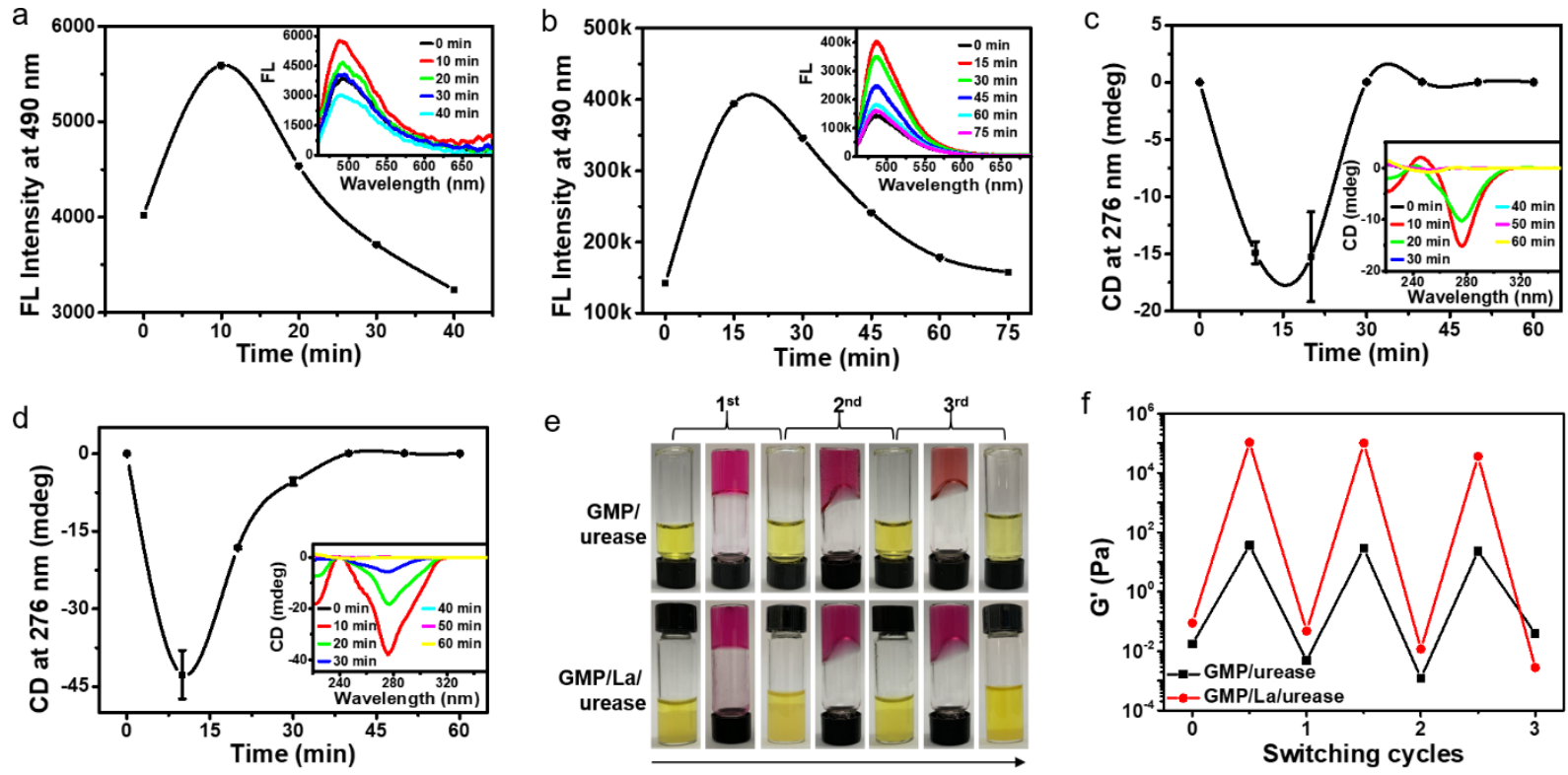

Fig. 3 (a, b) Time-dependent fluorescence intensity (at $490 \mathrm{~nm}$ ) changes of ThT-containing GMP/urease (a) and GMP/La/urease (b) systems after fuel addition. Insets show the fluorescence spectra at different time after fuel addition. (c, d) Time-dependent CD (at $276 \mathrm{~nm}$ ) changes of GMP/urease (c) and GMP/La/urease (d) systems after fuel addition. Insets show the CD spectra at different time after fuel addition. (e) Repeated sol-to-gel-to-sol and precipitate-to-gel-to-precipitate transitions of GMP/urease and GMP/La/urease systems, respectively. (f) The change in storage modulus $\left(\mathrm{G}^{\prime}\right)$ of GMP/urease and GMP/La/urease systems (initial $\mathrm{pH}$ 8) during repeated fuel-driven topology switching cycles.

On the basis of the above results, we propose a mechanism of topological reconfiguration of molecular assemblies for the nonequilibrium systems. Before fuel addition, the GMP/urease system presents weak alkalinity ( $\mathrm{pH} \mathrm{8),} \mathrm{GMP} \mathrm{and} \mathrm{partial} \mathrm{urease} \mathrm{self-assemble} \mathrm{into} \mathrm{irregular}$ shaped nanoparticles. In this condition, stacked GMP or G-quartets with a stacking distance of 
3.3 ̊̊ exist in the system, as evidenced by XRD (Fig. 2a). After addition of acidic urea solutions, the $\mathrm{pH}$ of the system declines rapidly to 4, and columnar G-quadruplexes with a diameter of 2.0 $\mathrm{nm}$ form by ion-dipole interactions, wherein the distance between two adjacent G-quartets is 3.3 $\AA$ A. In this condition, urease serves as a crosslinker to connect the intertwined 1D nanofibers to form a 3D network mainly based on electrostatic interactions (Fig. S7), which can be confirmed by the difference in phase behavior between the GMP solution and GMP/urease gel at $\mathrm{pH} 4$ (Fig. 2a and S5a). The stronger binding force between GMP and urease (greater binding constant, Fig. 2h) also confirm this. With the enzymatic hydrolysis of urea, the $\mathrm{pH}$ of the system gradually increases, and the crosslinked nanofibers recover to the irregular shaped nanoparticles. The addition of $\mathrm{La}^{3+}$ causes macroscopic precipitates to form before fuel addition because the multivalent $\mathrm{La}^{3+}$ can crosslink GMP and urease molecules. After fuel addition, columnar G-quadruplexes with a diameter of $2.0 \mathrm{~nm}$ form by $\mathrm{La}^{3+}$-dipole interactions, and the distance between two adjacent G-quartets is $3.4 \AA$. In this condition, urease still acts as a crosslinker to contribute to the formation of hydrogels. Lastly, the hydrogels return to precipitates because of fuel depletion. Notably, throughout the entire phase transition processes for both GMP/urease and GMP/La/urease systems, the molecules are in associating states, but the molecular assemblies undergo a topological morphology transformation. This fact implies that the conventional 2D nonequilibrium assembly (two dimensions: degree of aggregation and time) is moved to the third dimension (topological morphology of molecular assemblies). This change in dimension is the major feature of our nonequilibrium assembly mechanism, which is different 
from the conventional 2D nonequilibrium assembly in biological and synthetic systems. On the basis of this nonequilibrium assembly mechanism, we can accomplish the dynamic topological reconfiguration of molecular assemblies for programming supramolecular structures and, therefore, yield G-quartet-based transient supramolecular hydrogels with tunable lifetime.

Solid vessels have a long history of confining and storing liquids, but they are not adaptive and are difficult to remove. Liquid walls have attracted more and more attention because they are functional, adaptive and easy to remove and re-form. Common strategies to control liquids include patterning surfaces, embedding water into a matrix of viscous oil, manipulating water-oil emulsions in microfluidic devices, and holding aqueous channels by immiscible magnetic liquid barriers. ${ }^{36-40}$ Our G-quartet-based transient supramolecular hydrogels with good adaptivity and dissipative properties are promising transient wells for gel-templated polymerization. Rheological studies (Fig. 4a-f) indicated that both the GMP/urease and GMP/La/urease hydrogels had good self-healing ability, and the storage modulus generally increased with increasing GMP concentration on the premise of keeping a constant urease concentration and an identical ratio of GMP to $\mathrm{La}^{3+}$. The GMP/La/urease hydrogels showed poor injectability (Fig. 4f) because of their high stiffness. By contrast, GMP/urease hydrogels with good injectability could be used for writing English letters (Fig. 4g). Most importantly, the letters made from the transient hydrogels were self-erasable because of the pre-programmed liquefaction (Fig. 4g). The tendency to increase and then decrease $G^{\prime}$ made the transient hydrogels maintain a fixed shape at a set time, followed by turning into liquids that were easy to remove (Fig. 4h). The transient 
hydrogels could also be used for drawing closed loop shapes. To broaden the application range of transient hydrogels, we used the closed shapes of transient hydrogels as self-dissociative wells for transient-gel-templated polymerization (Fig. 5a). A closed gel wall (i.e., hydrogel well) was formed by injecting the GMP/urease active hydrogels (GMP concentration: $250 \mathrm{mM}$ ) onto a substrate without pretreatments. Afterward, a liquid monomer of poly(propylene glycol) diacrylate (PPGDA) and a photo-initiator of Irgacure 2959 were added into the hydrogel well, followed by irradiation with UV light (wavelength: $365 \mathrm{~nm}$ ) for 2 min to initiate polymerization (Fig. 5b). After polymerization, the transient hydrogels underwent pre-programmed liquefaction, and the liquid could be removed simply by a water rinse or suction, yielding a free-standing polymer membrane with a desired shape. Notably, the transient hydrogels with various shapes could be directly formed on diverse substrates, such as glass, poly(tetrafluoroethylene) (PTFE), and stainless steel. The property of forming different shapes on different substrates without pretreatment is a great advantage of the transient hydrogel wells. The scanning electron microscopy (SEM) image in Fig. 5c shows that the lateral view of the free-standing polymer membrane made by the transient-gel-templated polymerization was even and flat. Energy dispersive spectroscopy (EDS) indicated that there were no characteristic elements of GMP and urease in the free-standing polymer, which implied that GMP and urease in the transient hydrogels could not diffuse into the hydrophobic liquid monomers. However, the characteristic elements of GMP were found in the resulting polymer when a hydrophilic liquid monomer of poly(ethylene glycol) diacrylate (PEGDA) was used for the transient-hydrogel-templated 
polymerization (Fig. S10). Therefore, this method is more suitable for the polymerization of hydrophobic liquid monomers. The transient-gel-templated polymerization strategy is not limited by substrates and could expand the use of nonequilibrium hydrogel systems.
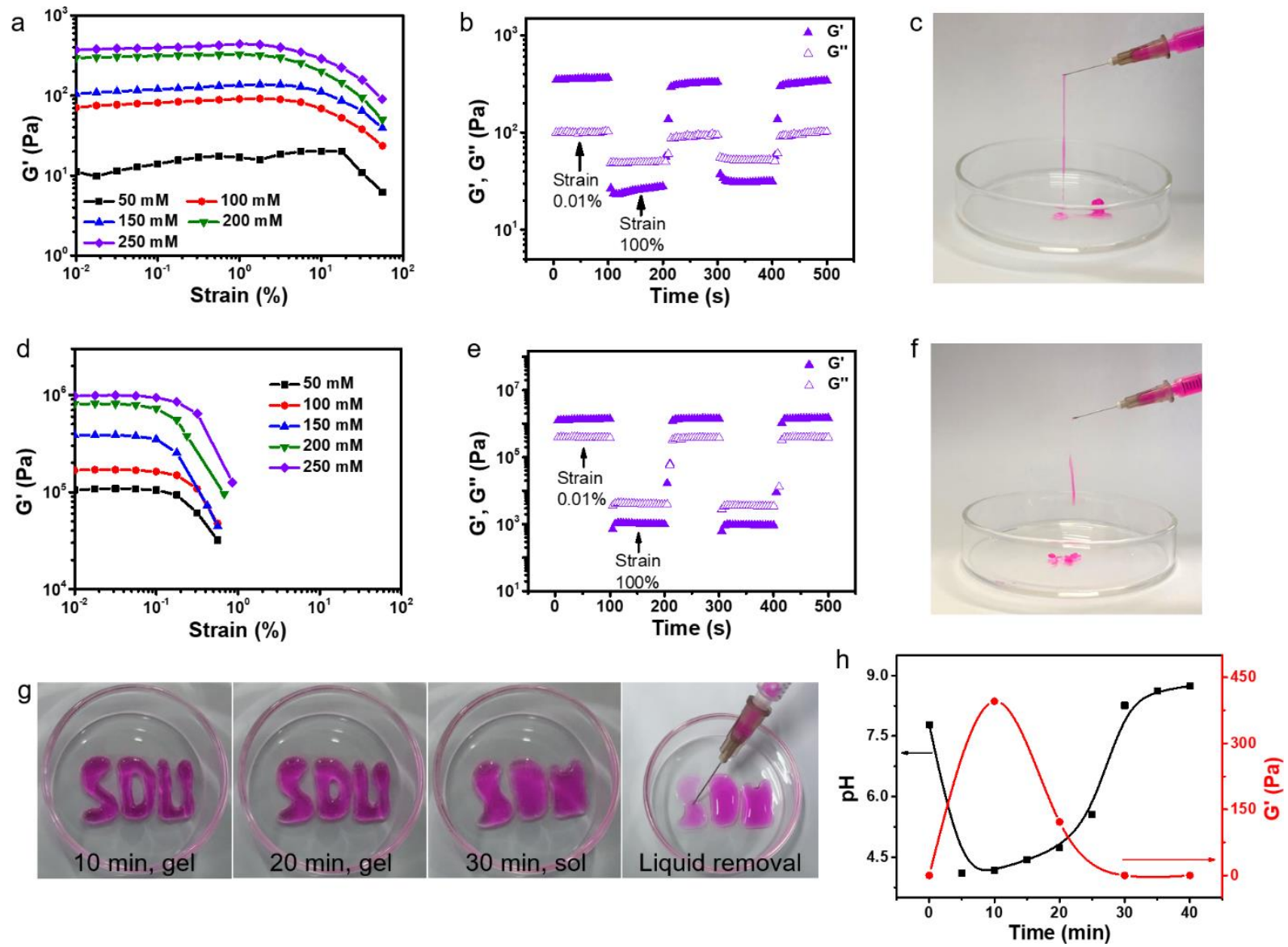

Fig. 4 (a) Strain sweep of GMP/urease hydrogels. (b, c) Thixotropic loop tests (b) and injection tests onto a glass substrate (c) of GMP/urease hydrogels $\left(C_{\mathrm{GMP}}=250 \mathrm{mM}\right)$. (d) Strain sweep tests of GMP/La/urease hydrogels. (e, f) Thixotropic loop tests (e) and injection tests onto a glass substrate (f) of $\mathrm{GMP} / \mathrm{La} /$ urease hydrogels $\left(C_{\mathrm{GMP}}=250 \mathrm{mM}\right)$. (g) Gel-to-sol transitions of $\mathrm{GMP} /$ urease system $\left(C_{\mathrm{GMP}}=250 \mathrm{mM}\right)$ mediated by enzymatic reaction. (h) Evolutions of $\mathrm{pH}$ and $\mathrm{G}^{\prime}$ during time sweeps for GMP/urease systems $\left(C_{\mathrm{GMP}}=250 \mathrm{mM}\right)$ mediated by enzymatic reaction. 

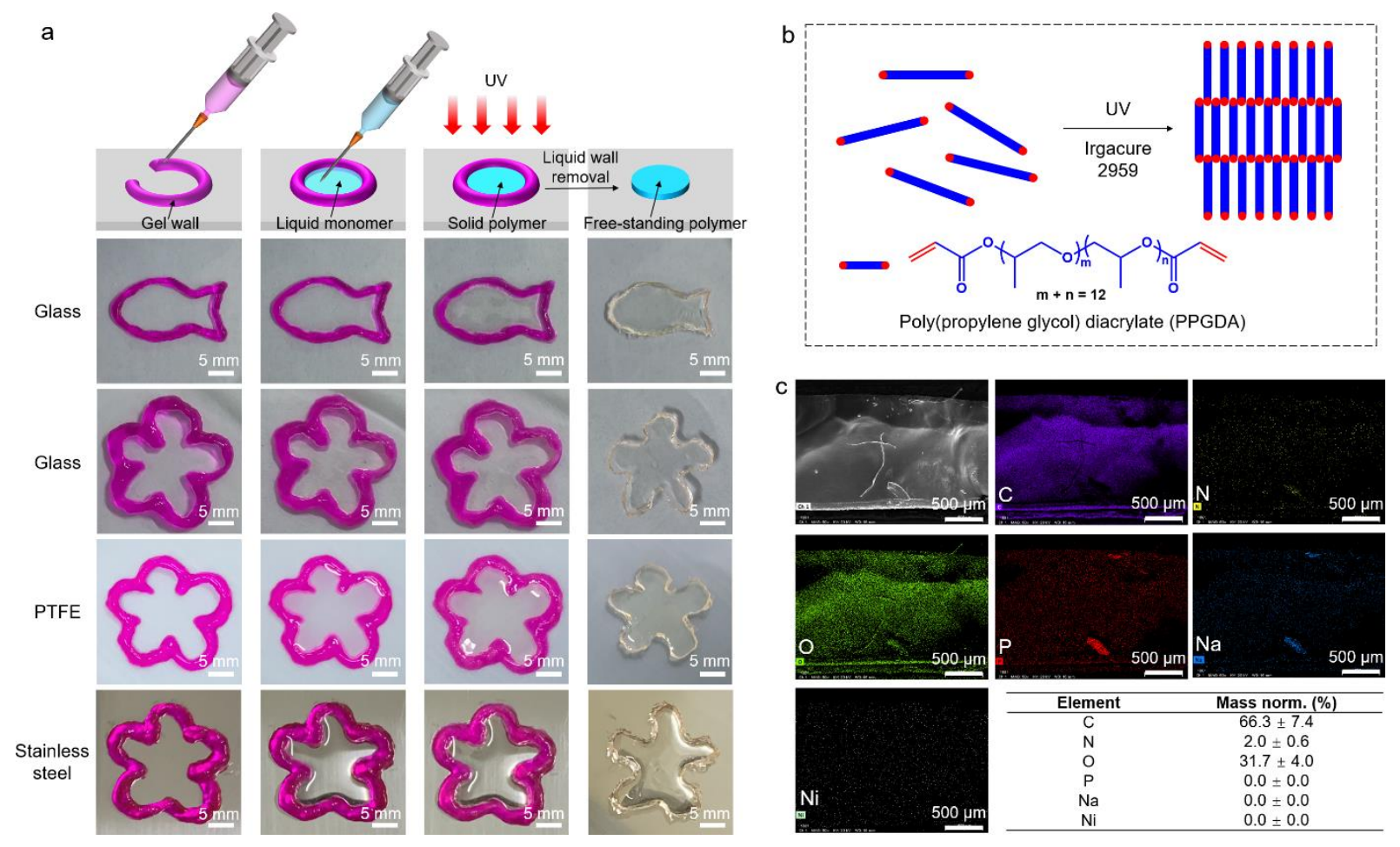

Fig. 5 (a) Schematic and photographs showing the transient-gel-templated polymerization of PPGDA on different substrates. (b) Schematic of the polymerization of PPGDA using Irgacure 2959 as a photo-initiator. (c) SEM and EDS mapping images of the lateral view of a free-standing polymer membrane prepared by transient-gel-templated polymerization of PPGDA, and a table summarizing the percent of each element.

Conductive hydrogels combine electrical conductivity and flexibility to mimic the mechanical and sensory functions of human skin, and have attracted great attention in the fields of wearables, implantable biosensors and artificial skin over the past few decades. ${ }^{41-45}$ With the rapid development of technology, transient electronics and ionotronics are becoming increasingly prevalent due to their advantages, such as adjustable life time and degradable property. ${ }^{46,47}$ The G-quartet-based transient supramolecular hydrogels in this work have a good ionic conductivity of $0.01 \mathrm{~S} / \mathrm{cm}$ (Fig. 6a) because of the large amount of salts in the system. The integration of excellent dissipative properties and ionic conductivity enables the nonequilibrium 
supramolecular hydrogels to serve as transient ionotronics. A transient supramolecular hydrogel was formed by adding chemical fuels into a GMP/urease solution in an eppendorf tube, and it was connected with a LED indicator in a series circuit having a $4 \mathrm{~V} \mathrm{AC} \mathrm{power} \mathrm{(Fig.} \mathrm{6b,} \mathrm{c).} \mathrm{The}$ LED indicator could be lit due to the effective contact between the hydrogel and wires. Afterward, the transient supramolecular hydrogel underwent pre-programmed liquefaction and the liquid flowed down to the bottom of the tube, the LED indicator became dim due to the loss of effective contact with the wires (Video 1). The lifetime of the transient ionotronics can be precisely controlled by varying the concentration of urea in the chemical fuel (Fig. 6d). This is a distinct feature that enables stable operation of the transient ionotronics over a predefined time frame.

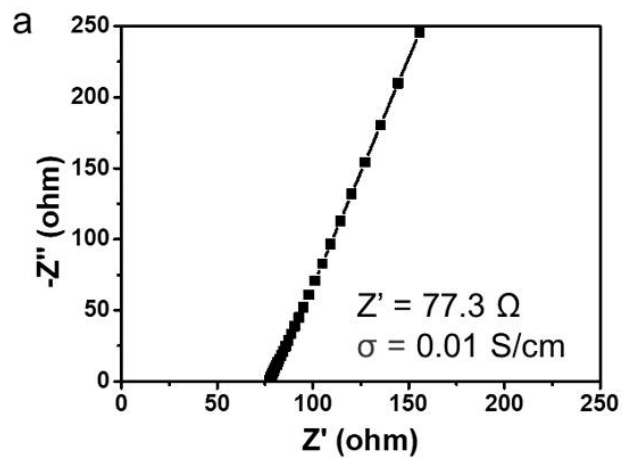

C

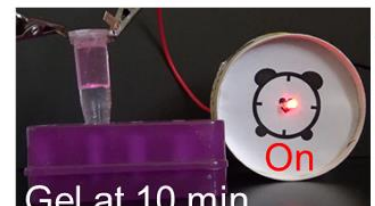

b
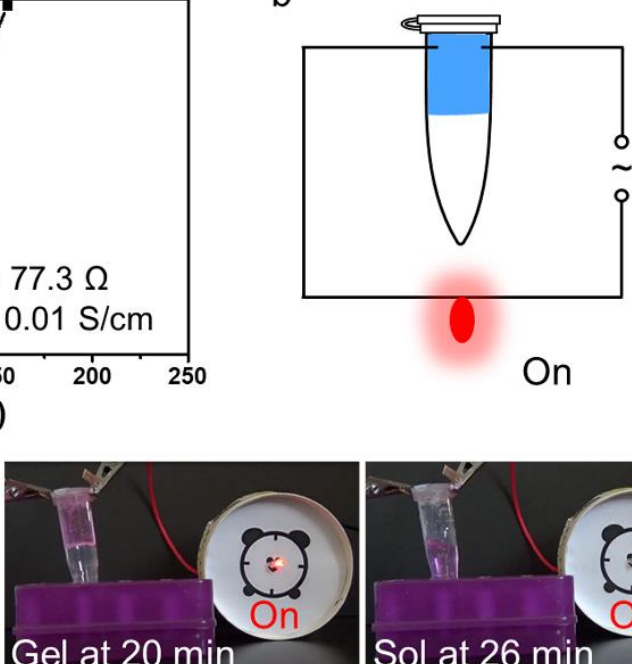

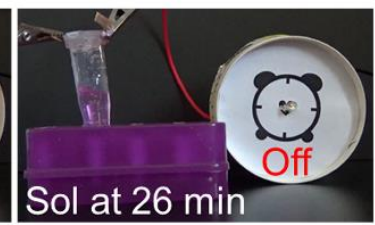

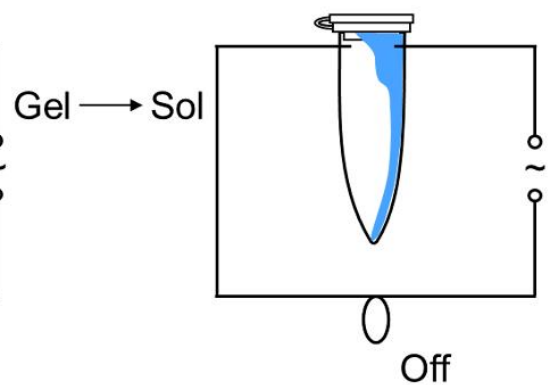

d

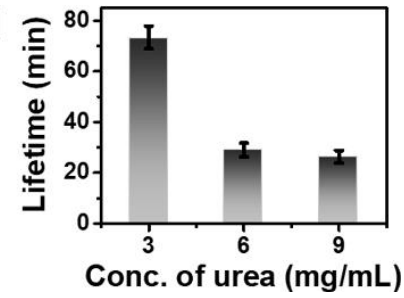

Fig. 6 (a) Electrochemical impedance spectrum of a GMP/urease transient hydrogel $\left(C_{\mathrm{GMP}}=250\right.$ $\mathrm{mM}$ ). (b) Schematic showing the working mechanism of transient ionotronics. (c) Sequential photographs recording the working process of the transient ionotronics at room temperature. (d) Lifetimes of transient ionotronics driven by addition of chemical fuels with different urea concentrations. 


\section{Discussion and conclusions}

The G-quartets stabilized by the formation of hydrogen bonds at the Hoogeteen face make guanosine and its derivatives highly interesting for the fundamental research of supramolecular assembly. ${ }^{23-25,27,48,49}$ The G-quartet-based supramolecular networks at equilibrium have long been regarded as critical platforms for assembly mechanism study and structure analysis. ${ }^{23,24}$ In contrast, the research on G-quartet-based nonequilibrium systems is rarely reported. Until recently, Li and coworkers developed the first transient supramolecular chiral G-quadruplex hydrogel with a tunable lifespan ranging from minutes to hours. ${ }^{50}$ They found that the dynamic formation and dissociation of the borate eater bond between guanosine and 5-fluorobenoxane driven by chemical fuels (KOH and 1,3-propanesultone) led to a precipitate-solution-gel-precipitate transition. Our similar finding, the dynamic precipitate-to-gel-to-precipitate transition mediated by fuel-driven and enzyme-regulated co-assembly of GMP, $\mathrm{La}^{3+}$, and urease, confirmed that observation. Notably, throughout the entire phase transition processes for GMP/urease and GMP/La/urease systems, the molecules are in associating states, but the molecular assemblies undergo a topological morphology transformation. We stressed a mechanism of topological reconfiguration of molecular assemblies at both microscopic and macroscopic levels. This mechanism of topological reconfiguration will increase understanding of extant nonequilibrium systems. Li and coworkers proposed that the transient G-quadruplex hydrogels with a tunable lifetime could provide attractive potentials for next-generation smart applications due to the rapid self-healability and injectability. ${ }^{50}$ The 
application in fluid confinements and transient electronics of the resultant transient hydrogels in our study confirmed the potential. Our experimental results, along with the existing literature, led us to provide a new approach for the design of next generation intelligent materials with self-adaptability and interactivity.

In conclusion, we suggested a mechanism of topological reconfiguration of molecular assemblies for understanding a representative type of nonequilibrium assembly behavior in artificial systems. We studied two $\mathrm{pH}$ feedback systems, GMP/urease and GMP/La/urease, that showed sol-to-gel-to-sol and precipitate-to-gel-to-precipitate dynamic transitions, respectively. The temporally controlled nanostructure evolutions and phase transitions affirmed the mechanism. The transient hydrogels exhibited good spatiotemporal controllability over their physicochemical properties, and they were qualified to serve as self-erasable wells and self-dissociative ionotronics for transient-gel-templated polymerization and transient circuit connection, respectively. This study demonstrated the wide gaps between diverse nonequilibrium assembly models in biological and synthetic systems. However, we do not value one class over another, instead, we suggest that each model has its own scope of application and rationale. We anticipate that this mechanism of topological reconfiguration will increase understanding of extant nonequilibrium systems and guide the design and development of novel soft materials with advanced functions.

Electronic supplementary information (ESI) available: All experimental procedures and 
supporting data (TEM images, XRD patterns, SAXS patterns, FTIR spectra, CD spectra, Zeta potential, transient-gel-templated polymerization of PEGDA, and transient conducting) are available in the ESI (PDF) and Video 1 (MP4).

\section{Authors contributions}

P.L. performed experiments and wrote original draft. A.S. and J.H. gave advices during performing the experiments and analyzing the data. X.W. designed and supervised the project, revised the manuscript and was responsible for funding acquisition. All authors commented on the manuscript.

\section{Conflicts of interest}

There are no conflicts to declare.

\section{Acknowledgements}

This research was supported by the National Natural Science Foundation of China (Grant No. 21975145). Prof. Li Zhang and Jialin Cui of China Agricultural University are thanked for helpful discussion.

\section{References}

1 R. Merindol, A. Walther, Chem. Soc. Rev., 2017, 46, 5588-5619. 
2 S. A. P. van Rossum, M. Tena-Solsona, J. H. van Esch, R. Eelkema, J. Boekhoven, Chem. Soc. Rev., 2017, 46, 5519-5535.

3 N. Singh, G. J. M. Formon, S. De Piccoli, T. M. Hermans, Adv. Mater., 2020, 32, 1906834.

4 K. Das, L. Gabrielli, L. J. Prins, Angew. Chem., Int. Ed., 2021, 60, 20120-20143.

5 M. Weißenfels, J. Gemen, R. Klajn, Chem, 2021, 7, 23-37.

6 J. Boekhoven, W. E. Hendriksen, G. J. M. Koper, R. Eelkema, J. H. van Esch, Science, 2015, 349, 1075-1079.

7 S. Panja, B. Dietrich, O. Shebanova, A. J. Smith, D. J. Adams, Angew. Chem., Int. Ed., 2021, 60, 9973-9977.

8 M. Jain, B. J. Ravoo, Angew. Chem., Int. Ed., 2021, 60, 21062-21068.

9 S. Maiti, I. Fortunati, C. Ferrante, P. Scrimin, L. J. Prins, Nat. Chem., 2016, 8, 725-731.

10 M. Cheng, C. Qian, Y. Ding, Y. Chen, T. Xiao, X. Lu, J. Jiang, L. Wang, ACS Mater. Lett., 2020, 2, 425-429.

11 A. Fischer, S. Lilienthal, M. Vázquez-González, M. Fadeev, Y. S. Sohn, R. Nechushtai, I. Willner, J. Am. Chem. Soc., 2020, 142, 4223-4234.

12 S. Dhiman, A. Jain, M. Kumar, S. J. George, J. Am. Chem. Soc., 2017, 139, 16568-16575.

13 J. Leira-Iglesias, A. Tassoni, T. Adachi, M. Stich, T. M. Hermans, Nat. Nanotechnol., 2018, 13, 1021-1027.

14 K. Jalani, S. Dhiman, A. Jain, S. J. George, Chem. Sci., 2017, 8, 6030-6036.

15 H. Ke, L.-P. Yang, M. Xie, Z. Chen, H. Yao, W. Jiang, Nat. Chem., 2019, 11, 470-477. 
16 L. Heinen, A. Walther, Chem. Sci., 2017, 8, 4100-4107.

17 S. Ahmed, A. Chatterjee, K. Das, D. Das, Chem. Sci., 2019, 10, 7574-7578.

18 P. Li, Y. Zhong, X. Wang, J. Hao, ACS Cent. Sci., 2020, 6, 1507-1522.

19 P. Li, Y. Xia, J. Hao, X. Wang, Macromolecules, 2020, 53, 2856-2863.

20 Y. Zhong, P. Li, J. Hao, X. Wang, ACS Appl. Mater. Interfaces, 2020, 12, 6471-6478.

21 Y. Zhong, T. Chen, X. Wang, ACS Appl. Bio Mater., 2020, 3, 4507-4513.

22 P. Li, J. Hao, X. Wang, ChemSystemsChem, 2021, 3, e2100016.

23 J. T. Davis, Angew. Chem., Int. Ed., 2004, 43, 668-698.

24 L. Stefan, D. Monchaud, Nat. Rev. Chem., 2019, 3, 650-668.

25 X. Li, A. Sánchez-Ferrer, M. Bagnani, J. Adamcik, P. Azzari, J. Hao, A. Song, H. Liu, R. Mezzenga, Proc. Natl. Acad. Sci. U. S. A., 2020, 117, 9832-9839.

26 J. Zhang, X. Li, X. Sun, A. Song, Y. Tan, J. Hao, Sci. China Chem., 2018, 61, 604-612.

27 D. Hu, J. Ren, X. Qu, Chem. Sci., 2011, 2, 1356-1361.

28 S. Panja, D. J. Adams, Chem. Eur. J., 2021, 27, 8928-8939.

29 H. Che, S. Cao, J. C. M. van Hest, J. Am. Chem. Soc., 2018, 140, 5356-5359.

30 M. M. Wrobel, T. Bánsági, S. K. Scott, A. F. Taylor, C. O. Bounds, A. Carranza, J. A. Pojman, Biophys. J., 2012, 103, 610-615.

31 Y.-L. Wu, F. He, X.-W. He, W.-Y. Li, Y.-K. Zhang, Spectrochim. Acta A, 2008, 71, 1199-1203.

32 Y.-Q. Wang, H.-M. Zhang, Spectrochim. Acta A, 2012, 96, 352-357. 
33 J. Lu, Y. Xue, K. Bernardino, N.-N. Zhang, W. R. Gomes, N. S. Ramesar, S. Liu, Z. Hu, T. Sun, A. F. d. Moura, N. A. Kotov, K. Liu, Science, 2021, 371, 1368-1374.

34 X.-M. Chen, X.-F. Hou, H. K. Bisoyi, W.-J. Feng, Q. Cao, S. Huang, H. Yang, D. Chen, Q. Li, Nat. Commun., 2021, 12, 4993.

35 S. Masiero, R. Trotta, S. Pieraccini, S. De Tito, R. Perone, A. Randazzo, G. P. Spada, Org. Biomol. Chem., 2010, 8, 2683-2692.

36 J. M. Scheiger, M. A. Kuzina, M. Eigenbrod, Y. Wu, F. Wang, S. Heißler, S. Hardt, B. Nestler, P. A. Levkin, Adv. Mater., 2021, 33, 2100117.

37 J. Wang, W. Gao, H. Zhang, M. Zou, Y. Chen, Y. Zhao, Sci. Adv., 2018, 4, eaat7392.

38 P. Dunne, T. Adachi, A. A. Dev, A. Sorrenti, L. Giacchetti, A. Bonnin, C. Bourdon, P. H. Mangin, J. M. D. Coey, B. Doudin, T. M. Hermans, Nature, 2020, 581, 58-62.

39 J. Forth, X. Liu, J. Hasnain, A. Toor, K. Miszta, S. Shi, P. L. Geissler, T. Emrick, B. A. Helms, T. P. Russell, Adv. Mater., 2018, 30, 1707603.

40 T. S. Kaminski, P. Garstecki, Chem. Soc. Rev., 2017, 46, 6210-6226.

41 K. Meyers, B. P. Lee, R. M. Rajachar, Gels, 2021, 7, 53.

42 V. R. Feig, H. Tran, M. Lee, Z. Bao, Nat. Commun., 2018, 9, 2740.

43 J. Wang, J. Yu, T. Wang, C. Li, Y. Wei, X. Deng, X. Chen, J. Mater. Chem. B, 2020, 8, 3341-3356.

44 Y. Guo, Z. Fang, G. Yu, Polym. Int., 2021, 70, 1425-1432.

45 C. Yang, Z. Suo, Nat. Rev. Mater., 2018, 3, 125-142. 
46 Y. Gao, Y. Zhang, X. Wang, K. Sim, J. Liu, J. Chen, X. Feng, H. Xu, C. Yu, Sci. Adv., 2017, 3, e1701222.

47 K. K. Fu, Z. Wang, J. Dai, M. Carter, L. Hu, Chem. Mater., 2016, 28, 3527-3539.

48 K. Hoogsteen, Acta Cryst., 1959, 12, 822-823.

49 L.-J. Yu, R. Pang, S. Tao, H.-T. Yang, D.-Y. Wu, Z.-Q. Tian, J. Phys. Chem. A, 2013, 117, 4286-4296.

50 X.-Q. Xie, Y. Zhang, Y. Liang, M. Wang, Y. Cui, J. Li, C.-S. Liu, Angew. Chem. Int. Ed., 2022, DOI: 10.1002/anie.202114471. 
Table of Contents Graphic

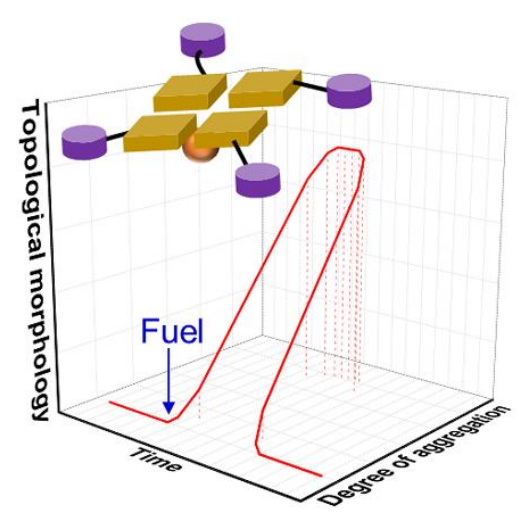

Transient hydrogel wall

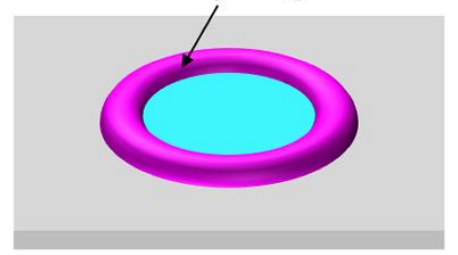

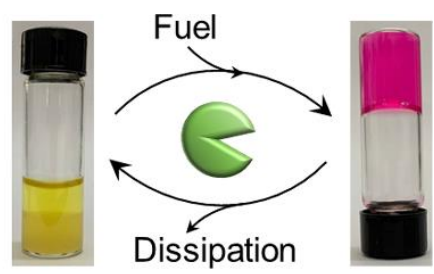

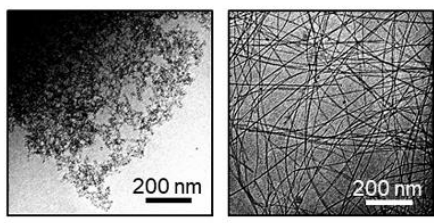

Transient ionotronic

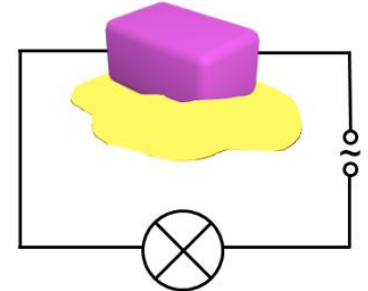

The G-quartet-based active supramolecular hydrogels were investigated, which enabled new insight into the nonequilibrium assembly mechanism. The active hydrogels hold great promise in applications such as fluid confinements and transient electronics. 